\title{
Consumo de alimentos ultraprocessados e o papel das escolas e da família na reeducação alimentar de adolescentes escolares
}

\author{
Consumption of ultraprocessed food and the role of schools and the family in the food re-education \\ of school adolescents
}

Consumo de alimentos ultraprocesados y el papel de las escuelas y la familia en la reeducación alimentaria de los adolescentes escolares

Recebido: 11/08/2021 | Revisado: 22/08/2021 | Aceito: 23/08/2021 | Publicado: 25/08/2021

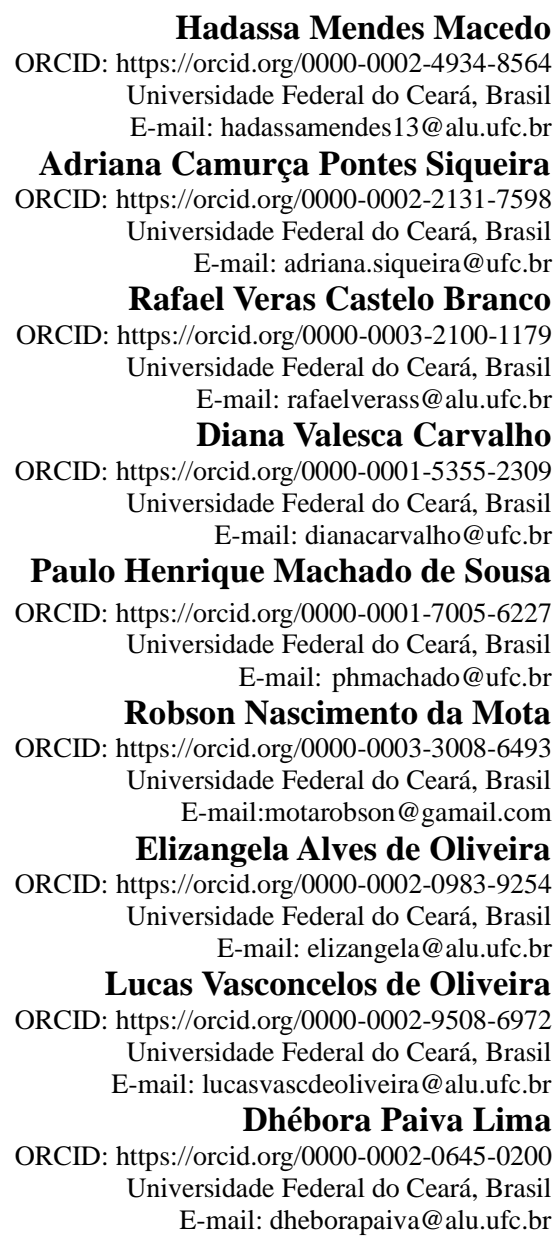

\section{Resumo}

Este artigo teve como objetivo apresentar, analisar e discutir dados sobre o consumo alimentar de escolares adolescentes e suas fontes de informações a respeito de saúde e alimentação e destacar a importância de uma alimentação saudável e do processo de reeducação alimentar de adolescentes por meio das escolas e de suas famílias. Para isso, foram realizadas pesquisas bibliográficas e de campo, com levantamento e análise de dados em um estudo transversal com 67 adolescentes escolares de idade entre 15 e 18 anos de uma escola pública de ensino médio de tempo integral. Aplicou-se um questionário validado contendo informações sobre consumo alimentar e fontes de informações sobre saúde e alimentação. A pesquisa mostrou que 59,16\% dos alunos pesquisados nunca ou quase nunca ingerem alimentos in natura, enquanto 43,86\% ingerem alimentos ultraprocessados, no mínimo, de duas a quatro vezes por semana. Isto é um dado preocupante, pois o não consumo adequado de alimentos in natura, aliado ao alto consumo de ultraprocessados é um fator de risco para o início de doenças crônicas não-transmissíveis. Por fim, destacou-se a importância dos pais e da escola como fonte de informação sobre saúde e alimentação, já que 68,09\% dos que tinham consciência sobre uma boa alimentação obtiveram informações em casa e 40,43\%, na escola. 
Recomenda-se novas pesquisas e políticas públicas voltadas para a Educação Alimentar e Nutricional, que garantam a segurança alimentar dessa população.

Palavras-chave: Alimentação; Saúde; Escola; Família; Adolescentes.

\begin{abstract}
This article aimed to present, analyze and discuss data on the food consumption of adolescent schoolchildren and their sources of information about health and nutrition and highlight the importance of healthy eating and the process of dietary re-education of adolescents through schools and of their families. For this, bibliographical and field research were carried out, with data collection and analysis in a cross-sectional study with 67 schoolchildren aged between 15 and 18 years old from a full-time public high school. A validated questionnaire containing information on food consumption and sources of information on health and nutrition was applied. The research showed that $59.16 \%$ of the students surveyed never or rarely eat fresh food, while $43.86 \%$ eat ultra-processed food at least two to four times a week. This is worrying, as the lack of adequate consumption of fresh foods, combined with the high consumption of ultra-processed foods, is a risk factor for the onset of non-transmissible chronic diseases. Finally, the importance of parents and the school as an information source on health and nutrition was highlighted, as $68.09 \%$ of those aware of good nutrition obtained information at home and $40.43 \%$ at school. New research and public policies aimed at Food and Nutrition Education are recommended, which guarantee the food security of this population.
\end{abstract}

Keywords: Food; Health; School; Family; Adolescents.

\title{
Resumen
}

Este artículo tuvo como objetivo presentar, analizar y discutir datos sobre el consumo de alimentos de los escolares adolescentes y sus fuentes de información sobre la salud y la alimentación y destacar la importancia de la alimentación saludable y el proceso de reeducación alimentaria de los adolescentes a través de las escuelas y sus familias. Para ello, se realizó una investigación bibliográfica y de campo, con recolección y análisis de datos en un estudio transversal realizado con 67 adolescentes escolares de entre 15 y 18 años de una escuela pública de tiempo completo, a través de la aplicación de un cuestionario validado que contiene información sobre el consumo de alimentos y fuentes de información sobre salud y alimentación. La investigación mostró que el $59.16 \%$ de los estudiantes encuestados nunca o casi nunca ingieren alimentos frescos, mientras que el $43.86 \%$ consume alimentos ultraprocesados al menos de dos a cuatro veces por semana. Este es un hecho preocupante, porque el no consumo adecuado de alimentos frescos, combinado con el alto consumo de alimentos ultraprocesados, es un factor de riesgo para la aparición de enfermedades crónicas no transmisibles. Finalmente, se destacó la importancia de los padres y la escuela como fuente de información sobre salud y alimentación, ya que el 68,09\% de los que conocían la buena alimentación obtuvieron información en casa y el 40,43\% en la escuela. Se recomiendan nuevas investigaciones y políticas públicas orientadas a la Educación Alimentaria y Nutricional, que garanticen la seguridad alimentaria de esta población.

Palabras clave: Alimentación; Salud; Escuela; Familia; Adolescentes.

\section{Introdução}

A cada ano é notado um aumento considerável no consumo de alimentos ultraprocessados por adolescentes e, como consequência, uma diminuição na ingestão de alimentos nutricionalmente mais ricos, como frutas, legumes e verduras. Tal fato é confirmado ao se analisar a Pesquisa de Orçamentos Familiares - POF - de 2017-2018, que aponta queda na frequência do consumo de alimentos saudáveis entre este público. Em contrapartida, o consumo de produtos como biscoitos recheados, pizzas, salgados fritos e assados, refrigerantes, macarrão instantâneo, sanduíches, entre outros, foi maior para essa população quando comparado a adultos e idosos (IBGE, 2020).

Outros estudos mostram que alimentos ultraprocessados, como os últimos citados acima e os fast-foods, ocasionam o aumento do LDL-colesterol (do inglês, low density lipoprotein), colesterol total, triglicerídeos e diminuição do HDL-colesterol (do inglês, high density lipoprotein), o que pode acarretar complicações na saúde do indivíduo, como sobrepeso, obesidade, hipertensão, cardiopatias e outras doenças crônicas (Beserra et al., 2020).

De acordo com a Organização Mundial da Saúde (do inglês, World Health Organization), o valor recomendado para o consumo de frutas, legumes e verduras é de $400 \mathrm{~g}$ por dia, equivalente a cinco porções de $80 \mathrm{~g}$, sendo esta ingestão uma das principais estratégias para proteção e prevenção a doenças crônicas não-transmissíveis, pois tais alimentos são fontes de vitaminas, minerais, fibras, entre outros nutrientes que auxiliam na manutenção da saúde do corpo humano (WHO, 2003; WHO, 2014). 
O Guia Alimentar para a População Brasileira também recomenda o consumo prioritário destes alimentos, os quais são categorizados em seu texto como in natura, à medida que são "obtidos diretamente de plantas e animais". O documento ainda orienta evitar os ultraprocessados, uma vez que são excessivamente calóricos e possuem uma composição nutricional desbalanceada, geralmente pelo alto índice de açúcar, sódio e aditivos de manipulação exclusivamente industrial (Brasil, 2014).

Diante disso, este artigo pretende abordar a problemática do visível aumento no consumo de alimentos ultraprocessados em detrimento da diminuição no consumo de alimentos saudáveis e de alto valor nutricional, tendo como principal objetivo apresentar, analisar e discutir dados sobre o consumo alimentar de escolares adolescentes e suas fontes de informações a respeito de saúde e alimentação, mostrando a relevância de um estreitamento das relações família-escola e da elucidação sobre uma alimentação adequada e saudável, e de como essas informações podem prevenir doenças e outras complicações à saúde desse grupo populacional.

\section{Metodologia}

O presente estudo, quanto aos objetivos, se caracteriza como exploratório, transversal e descritivo. Exploratório ao passo que reúne informações obtidas a partir da investigação, seleção e leitura de outras pesquisas, a exemplo de artigos científicos e levantamentos sociais, como a POF 2017-2018; transversal, por atravessar diversos campos do conhecimento, sejam eles a educação, a nutrição, a biologia, dentre outros; e descritivo, pois analisa, interpreta e classifica dados sem a interferência subjetiva do pesquisador ou mesmo dos avaliados (Andrade, 2010).

Quanto ao método, este pode ser considerado um estudo fenomenológico, uma vez que busca apresentar o fato como ele se apresenta, não ocorrendo interações com o contexto social, econômico, cultural, nem político, tendo cada fenômeno suas próprias características peculiares (Mota, 2009).

Em relação aos procedimentos técnicos, possui características de pesquisa bibliográfica, documental e de campo. Bibliográfica pelo estudo realizado a partir de informações não primárias, obtidas em livros, revistas e redes eletrônicas; documental pela utilização de informações primárias, documentos ainda sem o trato acadêmico e científico; e de campo pela necessidade de realizar parte da coleta de dados no local onde ocorre, ou ocorreu, o fenômeno, ou mesmo que dispõe de elementos para sua explicação (Mota, 2009), sendo utilizadas para o levantamento e análise de dados sobre o consumo alimentar de adolescentes escolares e suas fontes de informações sobre saúde e alimentação, como meio para obtenção dos resultados aqui mensurados.

A etapa bibliográfica e documental ocorreu através de busca ativa de artigos, livros, documentos e redes eletrônicas, entre os meses de setembro de 2020 e julho de 2021, no portal de periódicos da CAPES e nas bases de dados Scielo e Google Scholar, utilizando os descritores: "sobrepeso", "obesidade", "frutas", "legumes", "escolares", "saúde", "informações" e "adolescentes", considerando o intervalo de publicação das obras delimitado entre os anos de 2010 e 2021.

A pesquisa de campo, que possibilitou o conhecimento direto da realidade dos adolescentes escolares avaliados e a quantificação dos dados presentes neste estudo (Prodanov \& Freitas, 2013) ocorreu por meio da aplicação de questionário validado (Rodrigues, 2013), contendo interrogações sobre o consumo alimentar e as fontes de informações sobre saúde e alimentação, em uma amostra de 67 alunos do ensino médio ( $1^{\circ}$. ao $3^{\circ}$. ano), de ambos os sexos, com idades entre 15 e 18 anos, de uma escola pública de ensino médio de tempo integral do município de Fortaleza, no estado do Ceará. O questionário foi aplicado no período de março a junho de 2019.

Em relação à amostra, o objeto do estudo ou fornecimento de dados, trata-se de uma pesquisa não probabilística por acessibilidade, uma vez que a mesma não foi definida estatisticamente e os alunos e seus responsáveis participaram do estudo de maneira voluntária (Mota, 2009). 
Os alunos e seus responsáveis assinaram termos de consentimento livre e esclarecido, concordando em participar da pesquisa. Após a coleta, os dados foram digitados e analisados estatisticamente em planilha no Excel®.

\section{Resultados}

Por meio da aplicação do questionário e de sua posterior análise, percebeu-se que, ao serem indagados com as perguntas "você costuma comer frutas pelo menos cinco vezes por semana?" e "você costuma comer verduras ou legumes pelo menos cinco vezes por semana? (sem ser batata, inhame, mandioca)", a maioria dos alunos entrevistados relatou que não consumia frutas, bem como, legumes e verduras nessa frequência. (Figura 1).

Figura 1 - Porcentagem de alunos que não comem frutas, verduras e legumes 5x por semana.

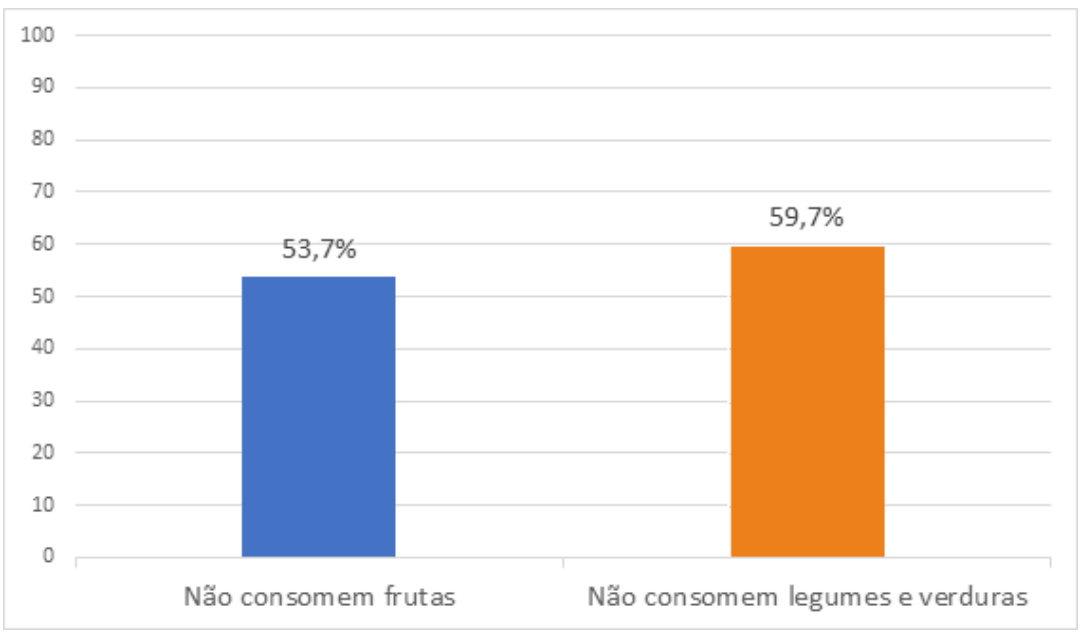

Fonte: Autores.

Os dados apresentados na Tabela 1 confirmam, portanto, a baixa frequência de consumo de frutas, verduras e legumes entre os adolescentes avaliados, visto que $59,16 \%$ deles afirmam "nunca ou quase nunca" consumir esses alimentos. 
Tabela 1 - Frequência de consumo de frutas, legumes e verduras pelo total de escolares (n=67) de uma escola pública de ensino médio de tempo integral em Fortaleza, CE.

Frutas,

Legumes e

Verduras

Alface

Repolho, couve ou acelga

Rúcula, almeirão ou agrião

Couve-flor ou brócolis

\section{Tomate}

Pepino

Chuchu

Maxixe ou Jiló

Abóbora ou

abobrinha

Cenoura

Beterraba

Quiabo ou

Vagem

Laranja ou

Tangerina

Banana

Mamão

Maçã

Melancia

Abacaxi

Manga

*Média de consumo por frequência

\section{Mais de De 2 a 3}

3 vezes por dia

0,00

0,00

1,49

8,96

0

0,00

0,00

0,00

0,00

0,00

0,00

0,00

0,00

8,96

11,94

1,49

5,97

7,46

10,45

1,49

$1,49 \quad 10,45$

19,4

7,46

22,38

10,45

5,97

7,46

13,43

11,94

8,96

5,97

5,97

10,45

5,58

0.94

4,25

7,47

6,06

5,97

4,48

0,00

16,42

14,93

7,46

11,94

4,48

10,45

5,97

8,96

7,71

10,45

11,94

1,49

13,43

10,45

8,96

13,43

8,96

8,96
De 1 a 3 vezes por mês

16,42

8,96

3,08

13,85

75,37

4,48

40,29

2,99

5,97

86,56

92,54

0,00

97,01

3,03

92,42

11,94

43,28

5,97

59,7

1,49

94,04

10,45

20,90

19,40

58,21

25,37

11,94

47,75

40,30

37,30

20,90

8,83

59,16

n: número da amostra.

* Média aritmética de cada frequência (coluna) de consumo de todos os alimentos, inclusive dos que apresentaram valor igual a 0 .

Fonte: Autores.

Por outro lado, foi considerada alta a frequência do consumo de alimentos ultraprocessados na amostra de estudantes pesquisados, conforme mostra a Tabela 2 . 
Tabela 2 - Frequência de consumo de ultraprocessados entre os escolares $(n=67)$ de uma escola pública de ensino médio de tempo integral em Fortaleza, CE.

\section{Frequência de consumo alimentar $(\%)$}

\begin{tabular}{|c|c|c|c|c|c|c|c|c|}
\hline $\begin{array}{l}\text { Alimentos } \\
\text { Ultraprocessados }\end{array}$ & $\begin{array}{l}\text { Mais de } \\
3 \text { vezes } \\
\text { por dia }\end{array}$ & $\begin{array}{l}\text { De } 2 \text { a } \\
3 \text { vezes } \\
\text { por dia }\end{array}$ & $\begin{array}{c}1 \text { vez } \\
\text { por dia }\end{array}$ & $\begin{array}{c}\text { De } 5 \text { a } 6 \\
\text { vezes } \\
\text { por } \\
\text { semana }\end{array}$ & $\begin{array}{c}\text { De } 2 \text { a } 4 \\
\text { vezes } \\
\text { por } \\
\text { semana }\end{array}$ & $\begin{array}{l}1 \text { vez por } \\
\text { semana }\end{array}$ & $\begin{array}{c}\text { De } 1 \text { a } 3 \\
\text { vezes por } \\
\text { mês }\end{array}$ & $\begin{array}{c}\text { Nunca ou } \\
\text { quase } \\
\text { nunca }\end{array}$ \\
\hline $\begin{array}{c}\text { Biscoito ou bolacha } \\
\text { doce }\end{array}$ & 5,97 & 11,94 & 16,42 & 10,45 & 25,37 & 11,94 & 1,49 & 16,42 \\
\hline $\begin{array}{c}\text { Biscoito ou bolacha } \\
\text { recheada }\end{array}$ & 5,97 & 10,45 & 17,91 & 8,96 & 17,91 & 19,39 & 4,48 & 14,93 \\
\hline Cachorro quente & 0,00 & 1,49 & 2,99 & 5,97 & 10,45 & 22,39 & 32,83 & 23,88 \\
\hline Linguiça & 1,49 & 5,97 & 4,48 & 11,94 & 11,94 & 23,88 & 22,39 & 17,91 \\
\hline $\begin{array}{c}\text { Frios (mortadela, } \\
\text { presunto, } \\
\text { apresuntado, } \\
\text { salame, salsicha) }\end{array}$ & 2,99 & 11,94 & 11,94 & 14,93 & 17,91 & 11,94 & 14,93 & 13,43 \\
\hline Pizza & 1,49 & 2,99 & 4,48 & 10,45 & 17,91 & 38,80 & 22,39 & 1,49 \\
\hline $\begin{array}{c}\text { Batata frita, chips } \\
\text { ou palha }\end{array}$ & 0,00 & 2,99 & 2,99 & 8,96 & 10,45 & 25,37 & 31,33 & 17,91 \\
\hline $\begin{array}{l}\text { Salgadinhos (do } \\
\text { tipo "cheetos", } \\
\text { "fofura", "torcida") }\end{array}$ & 1,54 & 4,62 & 7,69 & 7,69 & 10,77 & 29,23 & 13,85 & 24,61 \\
\hline $\begin{array}{l}\text { Salgadinhos (risole, } \\
\text { coxinha, pastel, } \\
\text { quibe, empada) }\end{array}$ & 2,99 & 4,48 & 5,97 & 13,43 & 16,42 & 32,83 & 16,42 & 7,46 \\
\hline Sorvete/Picolé & 1,52 & 0,00 & 4,55 & 9,09 & 10,61 & 27,27 & 28,78 & 18,18 \\
\hline Refrigerantes & 4,48 & 11,94 & 13,43 & 10,45 & 14,93 & 25,37 & 10,45 & 8,96 \\
\hline $\begin{array}{l}\text { Achocolatado em } \\
\text { pó (nescau, toddy) }\end{array}$ & 1,49 & 7,46 & 17,91 & 17,91 & 11,94 & 14,93 & 7,46 & 20,90 \\
\hline $\begin{array}{l}\text { Balas, caramelos, } \\
\text { chicletes, drops }\end{array}$ & 7,46 & 5,97 & 14,93 & 7,46 & 10,45 & 17,91 & 11,94 & 23,88 \\
\hline $\begin{array}{c}* \text { Média de consumo } \\
\text { por frequência }\end{array}$ & 2,88 & 6,33 & 9,67 & 10,59 & 14,39 & 23,17 & 16,83 & 16,14 \\
\hline
\end{tabular}

n: número da amostra.

* Média aritmética de cada frequência (coluna) de consumo de todos os alimentos, inclusive dos que apresentaram valor igual a 0.

Fonte: Autores.

Outros dados a serem destacados a partir da pesquisa são o acesso e as fontes de informações dos adolescentes escolares sobre alimentação saudável. Quando questionados se detinham conhecimento sobre o tema, a maioria respondeu que sim (Figura 2). 
Figura 2 - Porcentagem de alunos com conhecimentos sobre alimentação saudável.

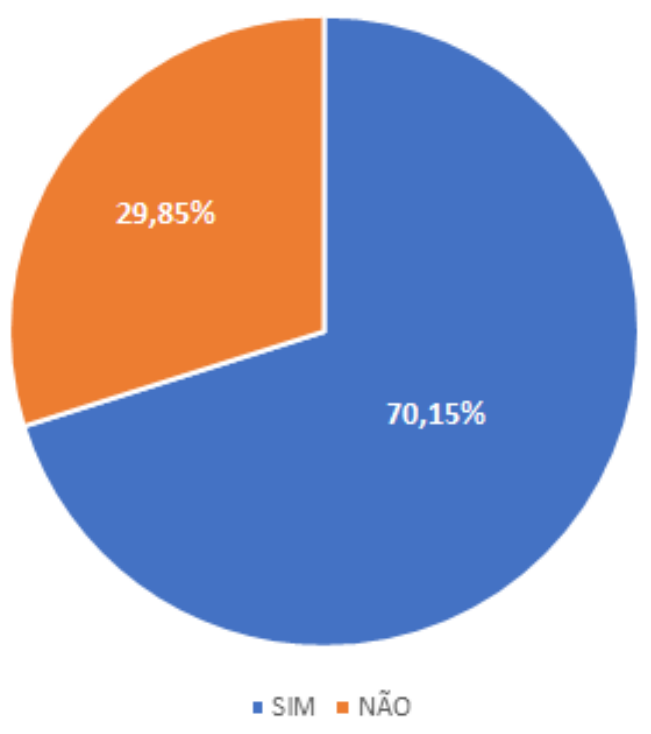

Fonte: Autores.

Dos adolescentes que relataram ter conhecimento sobre alimentação saudável, a maioria (68,09\%) respondeu que obteve esses conhecimentos em casa, com seus pais e/ou responsáveis, seguido pelos seus professores (Figura 3).

Figura 3 - Fonte de informação sobre alimentação saudável (\%).

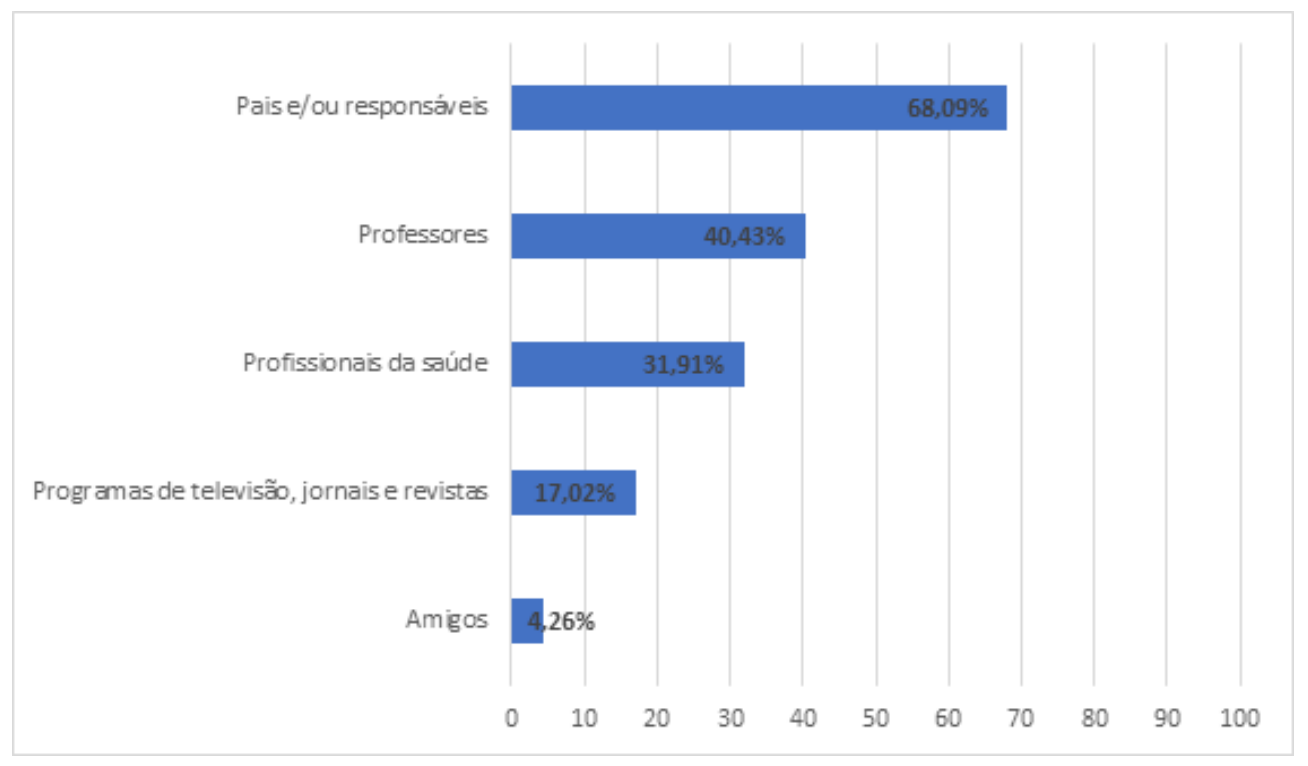

Fonte: Autores.

\section{Discussão}

O período da adolescência é conhecido por mudanças tanto psicológicas, como sociais e, principalmente, físicas. Nela, formam-se hábitos, pensamentos mais críticos e caráter. A alimentação está estritamente ligada a essas mudanças, delimitando condições favoráveis para o crescimento e a saúde do adolescente. Hábitos criados nesse período podem ser levados para toda a vida, sendo assim crucial a orientação de boas práticas alimentares para um bom desenvolvimento do indivíduo (Silva, Teixeira \& Ferreira, 2014). Os resultados demonstrados acima mostraram, no entanto, que a maioria dos adolescentes escolares avaliados não atingiu critérios desejáveis em relação à alimentação, a exemplo da recomendação da 
World Health Organization quanto ao consumo de 400g por dia de frutas, verduras e legumes, o equivalente a 5 porções de 80g diárias (WHO, 2003).

Como apresentado na Tabela 1, mais da metade dos adolescentes que participaram desta pesquisa relataram que sua frequência de consumo de legumes e verduras é "nunca ou quase nunca". Esse é um dado preocupante, visto que o não consumo de alimentos in natura, como os vegetais, ricos em fibras, vitaminas, minerais e compostos bioativos, pode ser um fator de risco para o surgimento de várias doenças crônicas não-transmissíveis (WHO, 2003). No Brasil, o Ministério da Saúde recomenda o consumo de tais alimentos como essencial para a prevenção de sobrepeso, obesidade e outras doenças crônicas como hipertensão, cardiopatias, diabetes, além de câncer, principalmente no aparelho digestivo, entre outras (Brasil, 2020).

Já na Tabela 2, os dados chamam atenção quando somados os valores das médias de frequência de consumo de alimentos ultraprocessados "mais de 3 vezes por dia", de "2 a 3 vezes por dia" e " 1 vez por dia", mostrando que 18,88\% dos adolescentes consomem ultraprocessados diariamente. Também é preocupante quando se observa a soma das médias de consumo semanal, de "5 a 6 vezes por semana", de " 2 a 4 vezes por semana" e "1 vez por semana", mostrando que $48,15 \%$ dos escolares consomem ultraprocessados semanalmente. A ingestão de ultraprocessados aliada ao não consumo adequado de alimentos in natura, principalmente os vegetais, como destacado anteriormente, pode se tornar um hábito desencadeador de várias doenças, como a obesidade, que vem aumentando consideravelmente na população estudada (Brasil, 2014).

Cabe destacar, de acordo com dados encontrados na literatura, que cerca de $60 \%$ dos adolescentes que possuem sobrepeso já antes da puberdade podem continuar em sobrepeso também na vida adulta, sendo esse um fator precursor ou agravante também de várias outras patologias (Noguera \& Hernández, 2012). O excesso de peso tem grande conexão com o desencadeamento de síndromes metabólicas e doenças crônicas que amplificam o risco de distúrbios pulmonares, ortopédicos, gastrointestinais, neuroendócrinos e consequências psicossociais graves (Machado, Feferbaum \& Leone, 2016). Desta forma, fica evidente a necessidade de uma diminuição no consumo dos alimentos ultraprocessados e um aumento no consumo dos considerados alimentos in natura ou minimamente processados na população estudada.

Ainda é possível observar, nos rótulos dos principais alimentos ultraprocessados consumidos pelos adolescentes aqui avaliados, uma quantidade, muitas vezes, excessiva de sódio, açúcar, gorduras, conservantes, corantes, aditivos, entre outras substâncias. No rótulo do biscoito/bolacha doce, de uma marca popular encontrada em supermercados do Brasil, por exemplo, $30 \mathrm{~g}$ de biscoito ( $71 \frac{1}{2}$ biscoitos) contém $135 \mathrm{kcal}, 19 \mathrm{~g}$ de carboidrato, $5,5 \mathrm{~g}$ de gordura e $59 \mathrm{mg}$ de sódio; já no rótulo de $30 \mathrm{~g}$ de biscoito recheado (3 biscoitos) da mesma marca, contém 20g de carboidrato, 5,5g gorduras, 70mg de sódio, além de corante caramelo IV. A salsicha, apontada na Tabela 2 com alta frequência de consumo pelos adolescentes, apresenta no rótulo de uma marca popular, que, em 50g, contém $123 \mathrm{kcal}, 9,9 \mathrm{~g}$ de gordura, $625 \mathrm{mg}$ de sódio, além de muitos aditivos, corantes e conservantes; já em 50g (5 fatias) de mortadela da mesma marca apresenta 161,5 kcal, 13,75g de gordura, 675mg de sódio, além de outras substâncias como estabilizantes, realçadores de sabor, corantes, conservantes e aromatizantes; e 40g (2,2 fatias) de presunto, também da mesma marca, contém 42,34 kcal, 1,2g de gordura, 464mg de sódio, além de outras substâncias, como reguladores de acidez, estabilizantes, aromatizantes, realçadores de sabor, conservantes e corantes.

Da mesma forma, uma das marcas líderes de refrigerantes consumida no Brasil e no mundo possui, em $350 \mathrm{~mL}, 149$ kcal, 37g de açúcar, 18mg de sódio, além de corante caramelo IV. E o achocolatado em pó, também da marca líder de mercado apresenta em sua maior concentração açúcar, em $20 \mathrm{~g}$ do produto, acrescido de $200 \mathrm{~mL}$ de leite integral, ele contém $198 \mathrm{kcal}$, $27 \mathrm{~g}$ de carboidrato, $25 \mathrm{~g}$ de açúcares, 6,7g de gordura, 95mg de sódio, entre outras substâncias como emulsificantes e aromatizantes.

Presente em vários alimentos, o corante caramelo IV é produzido a partir da combinação do açúcar com elementos químicos como ácidos e amônia, sob grande pressão e temperatura. Além da cor, ele também produz subprodutos prejudiciais à saúde, como por exemplo o 4-Metilimidazol (4-MI) (IDEC, 2019). Esse subproduto foi apontado pelo Programa Nacional de 
Toxicologia dos Estados Unidos como possível causador de câncer e, pela California Office of Environmental Health Hazard Assessment (2011), como cancerígeno, principalmente se ingerido mais de 29 microgramas por dia. Além disso, o estado estabeleceu que qualquer produto com concentração maior que 29 microgramas de 4-MI por porção deve informar o possível risco na embalagem. A quantidade desse elemento no Brasil não é informada nos produtos que o contém.

De acordo com a World Health Organization, o consumo excessivo de sódio e gordura, em especial a saturada, está diretamente associado com o risco de hipertensão e doenças cardiovasculares. Para reduzir esse risco, é recomendado o consumo de menos de 5 gramas de sal por dia, que equivale a 2.000 miligramas de sódio, e cerca de $30 \%$ de gordura do total de kcal ingeridas no dia, sendo, no máximo, 10\% de gorduras saturadas (WHO, 2014).

Outro ingrediente bastante utilizado nos alimentos ultraprocessados são os carboidratos refinados (simples). De acordo com a Food News Latam (2016), eles possuem alto índice glicêmico, sendo rapidamente absorvidos pelo organismo e, se consumidos em excesso, podem causar aumento de peso, levando à obesidade e, até mesmo, a mudanças metabólicas, doenças cardiovasculares, renais, hepáticas, diabetes, entre outras (Macedo, Aoyama, Silva \& Silva, 2019). Por isso, o consumo desses produtos deve ser evitado ao máximo, como orienta o Guia Alimentar para a População Brasileira (Brasil, 2014).

Um fator influenciador do hábito de consumir alimentos in natura é, portanto, o acesso a informações sobre seus benefícios à saúde. É de suma importância entender que frutas, verduras, legumes, grãos, sementes, castanhas e produtos saudáveis de origem animal, como ovo, leite, carnes brancas, que não passaram por processamento ou que tiveram um mínimo de alteração e de processamento possível, contêm micro e macro nutrientes e compostos bioativos que auxiliam na manutenção da saúde do corpo e são fundamentais para o crescimento e desenvolvimento dos seres humanos, principalmente de crianças e adolescentes (Brasil, 2014).

Alguns dos alimentos do grupo in natura que foram citados como nunca ou quase nunca consumidos na Tabela 1 são comumente achados durante todo o ano nos mercados e feiras do Brasil. Ao pesquisar na literatura suas composições, observamos que, por exemplo, o chuchu e a abobrinha apresentam vitaminas A, do complexo B e C. A abóbora, o pepino, a couve-flor, o brócolis, o repolho e a beterraba, além de conterem vitaminas $\mathrm{A}$, do complexo $\mathrm{B}$ e $\mathrm{C}$, também contêm vitamina $\mathrm{E}$ (Luengo, Parmagnani, Parente \& Lima, 2011). De acordo com a Tabela Brasileira de Composição de Alimentos (TACO), o mamão possui vitaminas A e C. Todos esses contêm fibras, cálcio, magnésio, manganês, fósforo, ferro, cobre, zinco, entre outras substâncias (NEPA, 2011).

Algumas vitaminas como a A, C e E auxiliam no cuidado com a pele, combatendo o envelhecimento precoce e o aparecimento de acne já na adolescência (Perssonelle, Pinto \& Ruiz, 1998). A vitamina D, muito conhecida por ser sintetizada através da pele, é também obtida por meio da alimentação, sendo de grande importância para a absorção de fósforo e cálcio pelo intestino, para a manutenção da saúde dos ossos, além de auxiliar na produção de insulina, prevenindo diabetes. Ela é encontrada mais comumente em peixes gordos (Alves et al., 2013). Já as vitaminas do complexo B, tão importantes para o nosso organismo, não são sintetizadas pelo mesmo, mas obtidas por meio de sua ingestão através dos alimentos, estando associada à produção de energia, ao bom funcionamento do metabolismo e de células nervosas e do cérebro (Rubert, Engel, Rohlfes, Marquardt \& Baccar, 2017).

Além das vitaminas, outras substâncias presentes em vários alimentos in natura e que trazem grandes benefícios para a saúde são as fibras e os minerais. As fibras auxiliam no bom funcionamento do trânsito intestinal, na redução dos níveis de colesterol total e LDL colesterol, na manutenção da glicemia, na redução de peso, no controle da pressão arterial e na prevenção de obesidade, também diminuindo os riscos de doenças cardiovasculares, câncer e diabetes tipo 2 (Bernaud \& Rodrigues, 2013). Já o cálcio, assim como o fósforo e o cobre, auxilia na manutenção e fortalecimento da estrutura óssea, trazendo um bom desenvolvimento ao corpo na fase da adolescência, também diminuindo os riscos de fratura (SBP, 2017). 
O magnésio, por sua vez, está envolvido em várias reações bioquímicas. Ele possui efeito antiarrítmico, colabora no metabolismo da glicose e na homeostase da insulina, e auxilia no crescimento ósseo; enquanto o ferro tem função no transporte de oxigênio e dióxido de carbono e também é parte ativa de enzimas no processo de respiração celular. Também faz parte da composição das proteínas hemoglobinas, que transportam o oxigênio do pulmão aos tecidos, e mioglobinas, que transportam e armazenam oxigênio no músculo, das enzimas citocromos, responsáveis pelo transporte de elétrons e degradação oxidativa de drogas, da catalase, encarregada da conversão de peróxido de hidrogênio em $\mathrm{O}_{2}$ e $\mathrm{H}_{2} \mathrm{O}$, das metaloproteínas (metabolismo oxidativo), do triptofano pirrolase (oxidação do triptofano), entre outros, sendo sua deficiência a causa mais comum para a anemia (Bueno \& Czepielewski, 2007; Cunha, Umbelino, Correia \& Neves, 2011).

Já o zinco está associado a uma gama de processos celulares como cofator para várias enzimas, tendo funções catalíticas, estruturais e reguladoras, influenciando a expressão gênica por meio de fatores de transcrição. A RNA polimerase, a transcriptase reversa e o fator de transcrição IIIA são exemplos de metaloenzimas que são dependentes de zinco. Ele é essencial na fase da adolescência por auxiliar no crescimento e, nessa fase, sua retenção aumenta significativamente, levando a um uso mais eficiente de fontes dietéticas. Sua deficiência pode causar lesões de pele, anorexia, retardo do crescimento, algumas alterações hormonais e na função imune, entre outros (Bueno \& Czepielewski, 2007).

Conhecer tudo isso é, portanto, essencial para a tomada de decisão quanto à mudança de hábitos alimentares, adotando práticas mais saudáveis. Um estudo realizado por Ribeiro, Menezes, Rosa e Costa (2019) apresenta fatores que influenciam a percepção dos escolares quanto à sua alimentação. Os voluntários adolescentes que participaram da referida pesquisa apontaram que estudam os conteúdos sobre alimentação disponíveis em seus materiais didáticos, como por exemplo, "as instruções dadas na pirâmide alimentar", sendo também orientado pelos professores "o consumo de alimentos saudáveis". Além disso, os dados também apontam a percepção dos estudantes quanto à alimentação oferecida pela escola, tratando alguns alimentos ofertados como saudáveis e outros como não saudáveis.

Neste estudo, alguns alunos comentaram a influência de suas famílias em sua alimentação. Apesar de relatarem a presença de arroz, feijão e outros alimentos minimamente processados, também foram apresentadas atitudes desfavoráveis por parte dos pais, como disponibilizar dinheiro para a compra de alimentos ultraprocessados para o lanche na escola. E, apesar da percepção sobre a alimentação saudável, os escolares não consumiam produtos in natura justificando tal atitude pelo sabor, segundo eles, desagradável do alimento saudável. Ao serem indagados sobre o porquê do baixo ou nenhum consumo de frutas, verduras e legumes, os alunos destacaram que isto era devido à falta de hábito e/ou por não gostarem, sendo que para frutas, em grande parte, era por falta de hábito e para verduras e legumes por gosto pessoal.

Monticelli, Souza e Souza (2012) destacam que os hábitos alimentares de crianças e adolescentes estão muito intimamente ligados à disponibilidade dos alimentos em seus domicílios. Essa disponibilidade, no entanto, estabelece relações com diversos fatores, entre eles: a renda e a escolaridade do (a) chefe da família ou do responsável pela aquisição dos referidos insumos, ou seja, às questões socioeconômicas. Castelo Branco et al. (2021) apontam, ainda, que a ausência da mulher na rotina domiciliar pode ser fator influenciador de casos de sobrepeso e obesidade entre adolescentes escolares. Em pesquisa realizada com alunos do $1^{\circ}$ ao $3^{\circ}$ ano do ensino médio de uma escola pública de tempo integral de Fortaleza, Ceará, os autores identificaram que a maioria dos avaliados diagnosticados com sobrepeso (53,8\%), obesidade (80\%) e obesidade grave (50\%) tinha sua família sustentada pela mãe, ou seja, a mulher se encontrava inserida do mercado de trabalho e ausente de casa durante a maior parte do dia. O estudo destaca ainda, que a escolaridade materna não teve influência na incidência dos quadros nutricionais citados e que os adolescentes que têm as mães presentes em sua rotina são os que mais consomem fast-foods.

A obesidade em adolescentes, todavia, não está ligada exclusivamente à disponibilidade e ao consumo excessivo de alimentos com alto valor calórico, mas também à redução de atividades físicas e ao concomitante aumento do tempo dedicado a videogames, redes sociais e televisão. Estudos apontam que o risco de obesidade entre adolescentes de 12 a 17 anos chega a 
crescer, em média, 2\% para cada hora adicional à frente da TV. Isso se deve porque atividades de lazer estáticas, sem movimento, tanto reduzem o gasto energético quanto incentivam o consumo de alimentos mais calóricos, seja por incentivo de comerciais ou estilos de vida. E, por outro lado, observa-se, à frente da televisão, a redução do consumo de frutas e verduras (Monticelli, Souza \& Souza, 2012).

Visto isso, e como reforçam os dados apontados pela Figura 2, a parceria entre a família e a escola é de grande importância para a promoção de hábitos saudáveis na vida dos adolescentes, já que a maior parte dos estudantes adquire informações sobre sua alimentação por meio dos pais e/ou professores.

O nível e a qualidade das informações absorvidas por adolescentes acerca do que seja uma alimentação saudável foi objeto de estudo realizado por Silva, Teixeira e Ferreira (2012), tomando como ponto de partida os hábitos alimentares desse público e a influência do seu meio social sobre sua alimentação. A pesquisa foi realizada com adolescentes escolares de 14 a 17 anos, e o que mais chama atenção é que os voluntários, na autoavaliação, ao responderem se relacionavam saúde à alimentação saudável, apontaram que, apesar de terem consciência de que não se alimentam bem e que não fazem nenhuma mudança em torno disso, ainda assim reconhecem a alimentação de qualidade como principal meio para a manutenção de uma boa saúde.

Isso demonstra que adolescentes têm conhecimento sobre a importância de hábitos alimentares saudáveis, embora não consigam colocá-los em prática, o que traz à tona inúmeros fatores que podem dificultar a tomada de decisão para mudança de determinadas práticas, como: o meio em que vivem, a cultura dos lanches rápidos, os chamados fast foods, o fácil acesso às comidas práticas e às lanchonetes e os vícios adquiridos desde a infância (Silva, Teixeira \& Ferreira, 2012), além da forte influência da mídia no incentivo de consumo de alimentos ultraprocessados (Melo, Lustoza, Ibiapina \& Landim, 2019).

Tudo isso aponta para a importância da Educação Alimentar e Nutricional desde a infância e durante a adolescência, sendo a escola um lugar de potencial transformação, à medida que, ao lado das famílias, é responsável pelo desenvolvimento do pensamento crítico de crianças e adolescentes. Uma ferramenta de Educação Alimentar e Nutricional (EAN) que vem sendo proposta na literatura é a inclusão da gastronomia como disciplina eletiva, ou mesmo da alimentação como tema transversal dentro do ambiente escolar. Tal ação pode tanto contribuir para o ensino interdisciplinar quanto para despertar o interesse de escolares pelo tema, além de incentivar a sua autonomia em torno de sua rotina e práticas alimentares. Castelo Branco et al (2021) destacam a importância da independência alimentar de adolescentes no sentido de garantir a Segurança Alimentar e Nutricional dessa população, retirando também da mulher, representada pela figura da mãe, a responsabilidade exclusiva pelo cuidado com a alimentação familiar.

Professores e pais, portanto, também devem ser frequentemente orientados sobre a importância do consumo de alimentos saudáveis, in natura, para se aperfeiçoarem como fontes dessas informações, uma vez que são, conforme apresentado na presente pesquisa, as principais fontes de informações sobre alimentação e saúde para os adolescentes estudados e para que, juntos aos adolescentes, consigam implementar processos efetivos de reeducação alimentar, inserindo hábitos saudáveis em seu dia-a-dia, não somente em relação à alimentação, mas também na prática de exercícios físicos, estando, assim, toda a família e a escola envolvidos de forma ativa nesse complexo processo de mudança de hábitos alimentares e estilo de vida saudável.

O Guia Alimentar para a População Brasileira pode contribuir também como outra importante ferramenta de EAN. Com uma linguagem simples e acessível, além de contar com uma versão especial para crianças, o documento, segundo Bezerra (2018, p.28), é "instigante não somente para o leitor especializado e profissionais das áreas de Segurança Alimentar e Nutricional (SAN) e EAN, mas para quaisquer pessoas interessadas por alimentação saudável, cultura alimentar e bem-estar em geral". 
Por fim, no caso das escolas de tempo integral, em que geralmente são oferecidas refeições aos alunos, as merendeiras e agentes de preparo dos alimentos exercem papel fundamental, pois, mesmo de forma indireta, ao selecionar e preparar alimentos, participam da educação alimentar dos escolares. Isso se dá, também, pela relação de expectativa e até mesmo dependência que se estabelece entre os estudantes e estes profissionais, como pode ser percebido pela fala de uma merendeira a partir do estudo realizado por Fernandes, Fonseca e Silva (2014):

Se faltamos e as preparações não podem ser totalmente elaboradas, os alunos são dispensados, mas se não tem professor, os alunos podem ser encaminhados a outras atividades no espaço escolar. O funcionamento da escola está associado diretamente ao fornecimento da alimentação. (p.44)

Isso se soma ao que é percebido na Figura 3, em que 40,43\% dos adolescentes afirmam obter conhecimento sobre alimentação saudável na escola. Dessa forma, torna-se indispensável que os governos municipais, estaduais e federal acompanhem o consumo alimentar dos escolares da rede pública, dando orientações, suporte e recursos aos gestores e cozinheiros/merendeiras, oferecendo também cursos de capacitação aos profissionais que trabalham na produção dos preparos ofertados, informando-os dos benefícios da diminuição do uso de ultraprocessados e do incentivo ao consumo de alimentos in natura.

\section{Considerações Finais}

A pesquisa destacou o consumo de alimentos ultraprocessados entre adolescentes, enfatizando que essa categoria de alimentos possui substâncias prejudiciais à saúde, bem como, o baixo consumo de produtos in natura, como frutas, verduras $\mathrm{e}$ legumes, ricos em macro e micronutrientes e compostos bioativos, importantes para manutenção da saúde, crescimento e desenvolvimento do corpo humano nessa fase da vida.

A maioria dos adolescentes possui conhecimentos sobre o que é uma alimentação saudável, apesar de não aplicar esses conhecimentos nos seus hábitos alimentares diários.

Outro fator de relevância determinado foi o papel dos pais e da escola no acesso e na construção do conhecimento dos adolescentes sobre sua própria alimentação, pois este grupo etário passa grande parte de seu tempo em casa ou no ambiente escolar, sendo esses familiares e profissionais seus guias e fortes influenciadores para que adolescentes entendam mais sobre a necessidade de bons hábitos, que os levem não somente a uma alimentação saudável, mas a uma vida saudável como um todo, prevenindo doenças como a obesidade, diabetes, cardiopatias, entre outras doenças crônicas.

Por fim, recomenda-se que novas pesquisas sejam realizadas, trazendo mais a fundo o porquê da diminuição do consumo de alimentos in natura e do aumento dos ultraprocessados entre adolescentes, já que estas ainda são escassas no Brasil. Sugere-se, ainda, que novas pesquisas e políticas públicas trabalhem metodologias de Educação Alimentar e Nutricional que possam auxiliar na mudança de hábitos e promover uma efetiva reeducação alimentar na população de adolescentes escolares brasileiros, auxiliando as escolas e famílias, com aplicação do Guia Alimentar para a População Brasileira e de oficinas de gastronomia nas escolas, que possibilitem mais autonomia alimentar entre adolescentes e contribua para a garantia da Segurança Alimentar e Nutricional nessa população.

\section{Referências}

Alves, M., Bastos, M., Leitão, F., Marques, G., Ribeiro, G. \& Carrilho, F. (2013). Vitamina D: importância da avaliação laboratorial. Revista Portuguesa de Endocrinologia, Diabetes e Metabolismo, 8 (1), 32-39. https://doi.org/10.1016/j.rpedm.2012.12.001

Andrade, M. M. de (2010). Introdução à Metodologia do Trabalho Científico (10a ed.). Editora Atlas S.A. 
Bernaud, F. S. R. \& Rodrigues, T. C. (2013) Fibra alimentar: ingestão adequada e efeitos sobre a saúde do metabolismo. Arquivos Brasileiros de Endocrinologia \& Metabologia, 57 (6), 397-405. https://doi.org/10.1590/S0004-27302013000600001

Beserra, J. B., Soares, N. I. da S., Marreiros, C. S., Carvalho, C. M. R. G. de, Martins, M. do C. de C. e, Freitas, B. de J. e S. de A., Santos, M. M. dos. \& Frota, K. de M. G. (2020). Crianças e adolescentes que consomem alimentos ultraprocessados possuem pior perfil lipídico? Uma revisão sistemática. Ciência \& Saúde Coletiva, 25(12), 4979-4989. https://doi.org/10.1590/1413-812320202512.29542018

Bezerra, J. A. B. (2018). Educação Alimentar e Nutricional: articulação de saberes. Edições UFC.

Brasil. (2014). Guia alimentar para a população brasileira (2a ed.). Ministério da Saúde, Secretaria de Atenção à Saúde, Departamento de Atenção Básica. https://bvsms.saude.gov.br/bvs/publicacoes/guia_alimentar_populacao_brasileira_2ed.pdf

Brasil. (2020). Plano de ações estratégicas para o enfrentamento das doenças crônicas e agravos não transmissíveis no Brasil 2021 -2030. Brasília - 2020. Ministério da Saúde. Secretaria de Vigilância em Saúde. Departamento de Análise em Saúde e Vigilância de Doenças Não-Transmissíveis. https://antigo.saude.gov.br/images/pdf/2020/October/01/Plano-DANT-vers--o-Consulta-p--blica.pdf

Bueno, A. L. \& Czepielewski, M. A. (2007). Micronutrientes envolvidos no crescimento. Revista Hospital de Clínicas Porto Alegre, 27(3), 47-56. https://www.lume.ufrgs.br/handle/10183/164794

California Office of Environmental Health Hazard Assessment (OEHHA). (2011). No significant risk level (nsrl) for the proposition 65 carcinogen 4 methylimidazole. California Environmental Protection Agency. https://oehha.ca.gov/proposition-65/chemicals/4-methylimidazole

Castelo Branco, R. V., Siqueira, A. C. P., Carvalho, D. V., Oliveira, E. A. de., Oliveira, L. V. de, \& Lima, D. P. (2021). Os impactos da inserção feminina no mercado de trabalho sobre o perfil nutricional e os hábitos alimentares de escolares adolescentes. Research, Society and Development, 10(7), e25010716586. https://doi.org/10.33448/rsd-v10i7.16586

Cunha, A. R., Umbelino, B., Correia, M. L. \& Neves, M. F. (2011). Efeitos do magnésio sobre a estrutura e função vascular. Revista Hospital Universitário Pedro Ernesto, 10(3), 39-45. https://www.e-publicacoes.uerj.br/index.php/revistahupe/article/view/8861

Fernandes, A. G. de S., Fonseca, A. B. C. da \& Silva, A. A. da. (2014). Alimentação escolar como espaço para educação em saúde. Ciência \& Saúde Coletiva, 19(1), 39-48. https://doi.org/10.1590/1413-81232014191.1711

Food News Latam. (2016) Carbohidratos simples Vs. Carbohidratos complejos. https://www.foodnewslatam.com/paises/4967-centro-am\%C3\%A9rica/5477carbohidratos-simples-vs-carbohidratos-complejos.html

IBGE - Instituto Brasileiro de Geografia e Estatística. (2020). Pesquisa de Orçamentos Familiares 2017-2018 - POF -: análise do consumo alimentar pessoal no Brasil. https://biblioteca.ibge.gov.br/visualizacao/livros/liv101742.pdf

IDEC - Instituto Brasileiro de Defesa do Consumidor. (2019). Você sabe o que é Caramelo IV?. https://idec.org.br/consultas/dicas-e-direitos/voce-sabe-o-quee-caramelo-iv\#: : text=O\%20caramelo\%20IV\%2C\%20um\%20dos,at\%C3\%A9\%20em\%20alimentos\%20para\%20c\%C3\%A3es

Luengo, R. de F. A., Parmagnani, R. M., Parente, M. R. \& Lima, M. F. B. F. (2011) Tabela de composição Nutricional das Hortaliças. (2. ed.) Brasilia: Embrapa. https://www.embrapa.br/documents/1355126/9124396/Tabela\%2BNutricional\%2Bde\%2BHortali\%25C3\%25A7as/d4ae0965-9e94-4f19-a20eb7721bdc1266

Macedo, W. de L. R., Aoyama, E. de A., Silva, J. J. V. da \& Silva, S. (2019). Influência hormonal do excesso de carboidratos refinados e do meio ambiente no avanço da obesidade. Revista Brasileira Interdisciplinar de Saúde, 1(1), 19-25. https://revistarebis.rebis.com.br/index.php/rebis/article/view/13

Machado, R. H. V., Feferbaum, R. \& Leone, C. (2016). Consumo de frutas no Brasil e prevalência de obesidade. Journal Of Human Growth And Development, 26 (2), 243-252. http://dx.doi.org/10.7322/jhgd.119293

Melo, J. da C. B. de, Lustoza, G. F., Ibiapina, D. F. N. \& Landim, L. A. dos S. R. (2019). Influência da mídia no consumo de alimentos ultraprocessados e no estado nutricional de escolares. Revista Eletrônica Acervo Saúde, (29), e1016. https://doi.org/10.25248/reas.e1016.2019

Monticelli, F. D. B., Souza, J. M. P. de \& Souza, S. B. (2012). Consumo alimentar por adolescentes e a relação com fatores socioeconômicos e atividades de lazer sedentárias. Revista Nutrire, 37(1), 64-77. https://docs.bvsalud.org/biblioref/2017/05/322064/artigo.pdf

Mota, R. N. da (2009). Introdução à metodologia da pesquisa científica. Recife: O autor.

Noguera, M. D. \& Hernández, E. B. (2012). Consumo de frutas y verduras en escolares como estrategia preventiva del sobrepeso y obesidad infantiles. Duazary, 9(2), 151-158. https://revistas.unimagdalena.edu.co/index.php/duazary/article/view/176

NEPA - Núcleo de estudos e pesquisas em alimentação. (2011). Tabela Brasileira de Composição de Alimentos - TACO - (4a ed.). https://www.cfn.org.br/wp-content/uploads/2017/03/taco_4_edicao_ampliada_e_revisada.pdf

Perssonelle, J, G., Pinto, E. B. de S. \& Ruiz, R. de O. (1998) Vitamina A Ácida, Vitamina E e Vitamina C Injetáveis no Tratamento de Necroses de Pele. Revista Brasileira de Cirurgia Plástica, 13(1), 17-30. http://www.rbcp.org.br/details/288/pt-BR/vitamina-a-acida--vitamina-e-e-vitamina-c-injetaveis-notratamento-de-necroses-de-pele

Prodanov, C. C. \& Freitas, E. C. de. (2013) Metodologia do Trabalho Científico: Métodos e Técnicas da Pesquisa e do Trabalho Acadêmico. (2. ed.) Novo Hamburgo: FEEVALE.

Ribeiro, I. L., Menezes, I. L. R., Rosa, J. G. da S. S. \& Costa, I. do C. C. (2019). Alimentação saudável: a percepção de escolares sobre si próprios. Revista de Salud Pública, 21(3), e454047. https://doi.org/10.15446/rsap.v21n3.54047

Rodrigues, P. R. M. (2013) Hábitos alimentares, estilo de vida e estado nutricional de adolescentes: um estudo de base escolar em Cuiabá - MT. Tese de doutorado, Programa de Pós-graduação em Nutrição, Instituto de Nutrição, Universidade Federal do Rio de Janeiro. Rio de Janeiro, Brasil. 
Research, Society and Development, v. 10, n. 11, e122101119338, 2021

(CC BY 4.0) | ISSN 2525-3409 | DOI: http://dx.doi.org/10.33448/rsd-v10i11.19338

Rubert, A., Engel, B., Rohlfes, A. L. B., Marquardt, L. \& Baccar, N. de M. (2017). Vitaminas do complexo B: uma breve revisão. Revista Jovens Pesquisadores, 7(1), 30-45. http://dx.doi.org/10.17058/rjp.v7i1.9332

SBP - Sociedade Brasileira de Pediatria. (2017). Como otimizar a ingestão de cálcio e o ganho de massa óssea em adolescentes. Guia Prático de Atualização. Departamento Científico de Adolescência. https://www.sbp.com.br/fileadmin/user_upload/Adolescencia_-_Ca_e_ganho_massa_ossea_002_.pdf

Silva, J. G. da, Teixeira, M. L. de O. \& Ferreira, M. de A. (2012). Alimentação e saúde: sentidos atribuídos por adolescentes. Escola Anna Nery Revista de Enfermagem, 16(1), 88-95. https://doi.org/10.1590/S1414-81452012000100012

Silva, J. G. da, Teixeira, M. L. de O. \& Ferreira, M. de A. (2014). Alimentação na adolescência e as relações com a saúde do adolescente. Texto \& Contexto Enfermagem, 23(4), 1095-1103. https://www.scielo.br/scielo.php?script=sci_arttext\&pid=S0104-07072014000401095\&lng=en\&nrm=iso\&tlng=pt

WHO - World Health Organization. (2003) Diet, nutrition and the prevention of chronic diseases: report of a joint WHO/FAO expert consultation. WHO. https://apps.who.int/iris/bitstream/handle/10665/42665/WHO_TRS_916.pdf?sequence=1

WHO - World Health Organization. (2014) GLOBAL STATUS REPORT on noncommunicable diseases 2014. WHO. https://apps.who.int/iris/bitstre am/handle/10665/148114/9789241564854_eng.pdf;jsessionid=0DDAD83DCFACE57314190D41CF982EBD?sequence=1 